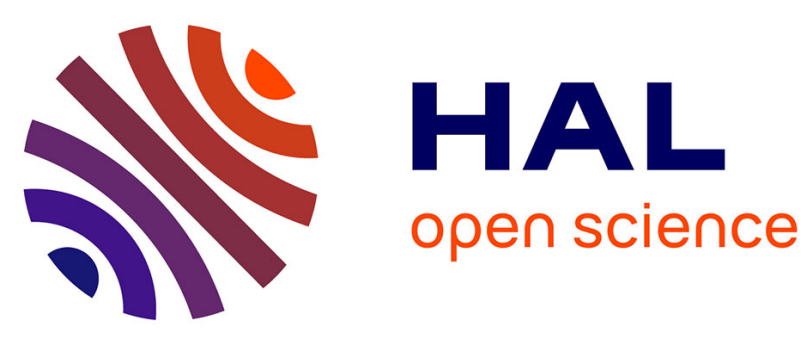

\title{
On polynomial Torus Knots
}

Pierre-Vincent Koseleff, Daniel Pecker

\section{To cite this version:}

Pierre-Vincent Koseleff, Daniel Pecker. On polynomial Torus Knots. Journal of Knot Theory and Its Ramifications, 2008, 17 (12), pp.1525-1537. 10.1142/S0218216508006713 . hal-00108510v2

\section{HAL Id: hal-00108510 https://hal.science/hal-00108510v2}

Submitted on 18 Oct 2007

HAL is a multi-disciplinary open access archive for the deposit and dissemination of scientific research documents, whether they are published or not. The documents may come from teaching and research institutions in France or abroad, or from public or private research centers.
L'archive ouverte pluridisciplinaire HAL, est destinée au dépôt et à la diffusion de documents scientifiques de niveau recherche, publiés ou non, émanant des établissements d'enseignement et de recherche français ou étrangers, des laboratoires publics ou privés. 


\title{
On polynomial Torus Knots
}

\author{
P. -V. Koseleff, D. Pecker \\ Université Pierre et Marie Curie \\ 4, place Jussieu, F-75252 Paris Cedex 05 \\ e-mail: $\{$ koseleff, pecker\}@math.jussieu.fr
}

October 19, 2007

\begin{abstract}
We show that no torus knot of type $(2, n), n>3$ odd, can be obtained from a polynomial embedding $t \mapsto(f(t), g(t), h(t))$ where $(\operatorname{deg}(f), \operatorname{deg}(g)) \leq(3, n+1)$. Eventually, we give explicit examples with minimal lexicographic degree.
\end{abstract}

keywords: Knot theory, polynomial curves, torus knots, parametrized space curve

Mathematics Subject Classification 2000: 14H50, 12D10, 26C10, 57M25

\section{Introduction}

The study of non compact knots began with Vassiliev Va type can be obtained from a polynomial embedding $t \mapsto(f(t), g(t), h(t)), t \in \mathbb{R}$. The proof uses Weierstrass approximation theorem on a compact interval, the degrees of the polynomials may be quite large, and the plane projections of the polynomial knots quite complicated.

Independently, Shastri [Sh] gave a detailed proof of this theorem, he also gave simple polynomial parametrizations of the trefoil and of the figure eight knot.

This is what motivated A. Ranjan and Rama Shukla [RS] to find small degree parametrizations of the simplest knots, the torus knots of type $(2, n), n$ odd, denoted by $K_{n}$. They proved that these knots can be attained from polynomials of degrees $(3,2 n-2,2 n-1)$. In particular, they obtain a parametrization of the trefoil $K_{3}$ analogous to Shastri's one. They also asked the natural question which is to find the minimal degrees of the polynomials representing a general torus knot of a given type (there is an analogous question in Vassiliev's paper Va ).

The number of crossings of a plane projection of $K_{n}$ is at least $n$ (Bankwitz theorem, see [Re]). It is not difficult to see, using Bézout theorem, that this plane curve cannot be parametrized by polynomials of degrees smaller than $(3, n+1)$.

Naturally, Rama Mishra (Mi]) asked whether it was possible to parametrize the knot $K_{n}$ by polynomials of degrees $(3, n+1, m)$ when $n \equiv 1$, or $0 \bmod 3$.

In this paper, we shall prove the following result

Theorem. If $n \neq 3$ is odd, the torus knot $K_{n}$ cannot be represented by polynomials of degrees $(3, n+1, m)$.

Our method is based on the fact that all plane projections of $K_{n}$ with the minimal number $n$ of crossings have essentially the same diagram. This is a consequence of the now solved classical 
Tait's conjectures [Mu, $\mathrm{Ka}, \mathrm{Pr}, \mathrm{MT}]$. This allows us to transform our problem into a problem of real polynomial algebra.

As a conclusion, we give explicit parametrizations of $K_{3}, K_{5}$ and $K_{7}$. By our result, they are of minimal degrees. We also give an explicit parametrization of $K_{9}$ with a plane projection possessing the minimal number of crossing points. This embedding is of smaller degree than those already known.

\section{The principal result}

If $n$ is odd, the torus knot $K_{n}$ of type $(2, n)$ is the boundary of a Moebius band twisted $n$ times (see $[\mathrm{Re}, \mathrm{Ka}, \mathrm{St}]$ ). The recently proved Tait's conjectures allow us to characterize plane projections

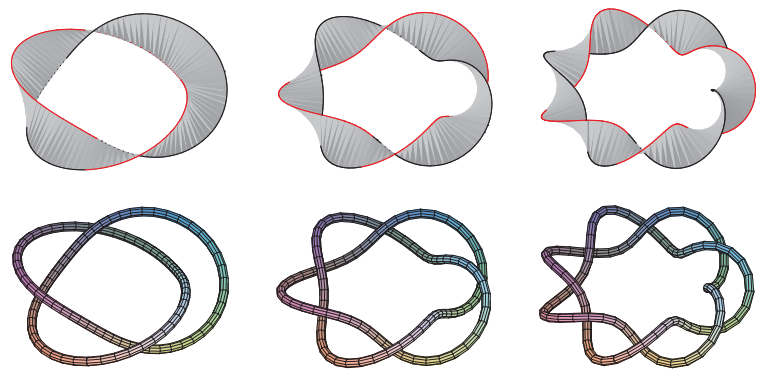

Figure 1: $K_{n}, n=3,5,7$.

of $K_{n}$ with the minimal number of crossings.

Lemma 1 Let $\mathcal{C}$ be a plane curve with $n$ crossings parametrized by $\mathcal{C}(t)=(x(t), y(t))$. If $\mathcal{C}$ is the projection of a knot $K_{n}$ then there exist real numbers $s_{1}<\cdots<s_{n}<t_{1}<\cdots<t_{n}$, such that $\mathcal{C}\left(s_{i}\right)=\mathcal{C}\left(t_{i}\right)$.

Proof. Let $\mathcal{C}$ be a plane projection of a knot of type $K_{n}$ with the minimal number $n$ of crossings.

Using the Murasugi's theorem B ([Mu] $)$ which says that a minimal projection of a prime alternating knot is alternating, we see that $\mathcal{C}$ is alternating.

Then the Tait's flyping conjecture, proved by Menasco and Thistlethwaite ([MT, $\mathbb{P r}])$, asserts that $\mathcal{C}$ is related to the standard diagram of $K_{n}$ by a sequence of flypes. Let us recall that a flype is a transformation most clearly described by the following picture.
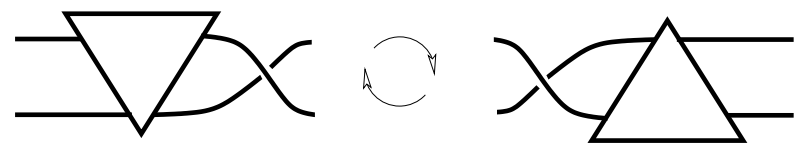

Figure 2: A flype

The standard diagram $\mathcal{S}_{0}$ of $K_{n}$ has the property ( $\mathrm{cf}[\mathrm{Re} \|)$ that there exist real numbers $s_{1}<\cdots<$ $s_{n}<t_{1}<\cdots<t_{n}$ such that $\mathcal{S}_{0}\left(s_{i}\right)=\mathcal{S}_{0}\left(t_{i}\right)$. It is alternating.

Let $\mathcal{S}$ be a diagram with real parameters $s_{1}<\cdots<s_{n}<t_{1}<\cdots<t_{n}$ such that $\mathcal{S}\left(s_{i}\right)=\mathcal{S}\left(t_{i}\right)$, and let us perform a flype of a part $B$ of $\mathcal{S}$

For any $(a, b, c) \in A \times B \times C$ we have

$$
s_{a}<s_{b}<s<s_{c}<t_{a}<t_{b}<t<t_{c} .
$$




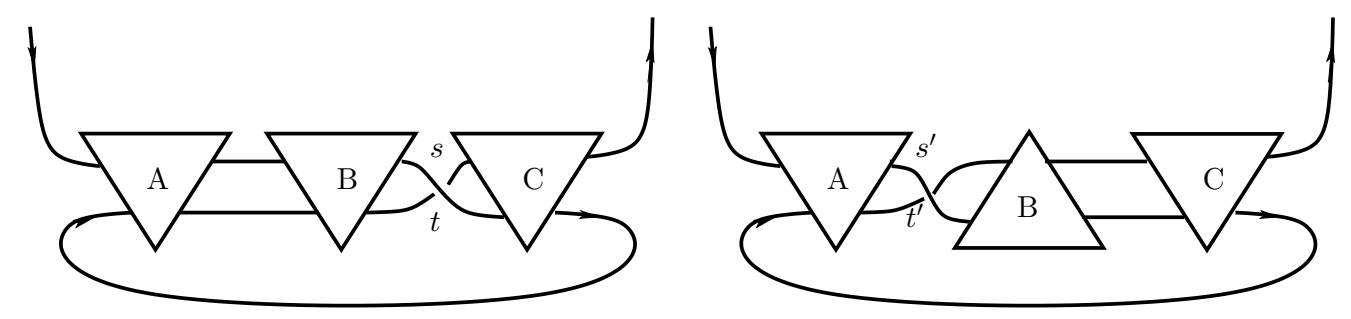

Figure 3: Flype on the part $B$

After the flype on $B$, we have new parameters corresponding to the crossing points satisfying

$$
s_{a}^{\prime}<s^{\prime}<s_{b}^{\prime}<s_{c}^{\prime}<t_{a}^{\prime}<t^{\prime}<t_{b}^{\prime}<t_{c}^{\prime} .
$$

The transformed diagram $\mathcal{S}^{\prime}$ has the same property: there exist real parameters $s_{1}<\cdots<s_{n}<$ $t_{1}<\cdots<t_{n}$, such that $\mathcal{S}^{\prime}\left(s_{i}\right)=\mathcal{S}^{\prime}\left(t_{i}\right)$.

So then, after any sequence of flypes, the transformed diagram will have the same property.

In this paper we shall consider polynomial knots, that is to say, polynomial embeddings $\mathbb{R} \longrightarrow$ $\mathbb{R}^{3}, t \mapsto(x(t), y(t), z(t))$. Polynomial knots are non-compact subsets of $\mathbb{R}^{3}$. The closure of a polynomial knot in the one point compactification $\mathbf{S}^{3}$ of the space $\mathbb{R}^{3}$ is an ordinary knot (see Va, Sh, RS] and figures at the end).

Lemma 2 Let $\mathcal{C}$ be a plane polynomial curve with $n$ crossings parametrized by $\mathcal{C}(t)=(x(t), y(t))$. Suppose that $\mathcal{C}$ is the projection of $K_{n}$ and $\operatorname{deg} x(t) \leq \operatorname{deg} y(t)$. Then we have $\operatorname{deg} x(t) \geq 3$. If $\operatorname{deg} x(t)=3$, then $\operatorname{deg} y(t) \geq n+1$.

Proof. $x(t)$ must be non-monotonic, so $\operatorname{deg} x(t) \geq 2$. Suppose that $x(t)$ is of degree 2. Then $x\left(t_{i}\right)=x\left(s_{i}\right)$ implies that $t_{i}+s_{i}$ is constant, and so the parameter values corresponding to the crossing points are ordered as

$$
s_{1}<\cdots<s_{n}<t_{n}<\cdots<t_{1} .
$$

We have a contradiction according to lemma 1

Suppose now that $\operatorname{deg} x(t)=3$. The crossing points of the curve $\mathcal{C}$ correspond to parameters $(s, t)$, $s \neq t$, that are common points of the curves of degrees 2 and $\operatorname{deg} y(t)-1$ :

$$
\frac{x(t)-x(s)}{t-s}=0, \frac{y(t)-y(s)}{t-s}=0
$$

By Bézout theorem $([\mathrm{Fi}])$, the number of such points are at most $2 \times(\operatorname{deg} y(t)-1) .(s, t)$ and $(t, s)$ are distinct points and correspond to the same crossing point. So, the curve $\mathcal{C}$ has at most $\operatorname{deg} y(t)-1$ crossing points, and this implies that $\operatorname{deg} y(t) \geq n+1$.

\section{Proof of the main result}

Our proof makes use of Chebyshev (monic) polynomials. 


\subsection{Chebyshev Polynomials}

Definition 1 If $t=2 \cos \theta$, let $T_{n}(t)=2 \cos (n \theta)$ and $V_{n}(t)=\frac{\sin ((n+1) \theta)}{\sin \theta}$.

Remark $1 T_{n}$ and $V_{n}$ are both monic and have degree $n$. We have

$$
V_{0}=1, \quad V_{1}=t, \quad V_{n+1}=t V_{n}-V_{n-1} .
$$

We have also

$$
T_{0}=2, \quad T_{1}=t, \quad T_{n+1}=t T_{n}-T_{n-1} .
$$

For $n \geq 2$, let $V_{n}=t^{n}+a_{n} t^{n-2}+b_{n} t^{n-4}+\cdots$. Using recurrence formula 1 , we get

$$
a_{n+1}=a_{n}-1, b_{n+1}=b_{n}-a_{n-1}
$$

so by induction,

$$
V_{n}=t^{n}-(n-1) t^{n-2}+\frac{1}{2}(n-2)(n-3) t^{n-4}+\cdots .
$$

We shall also need the following lemmas which will be proved in the next paragraph.

Lemma A. Let $s \neq t$ be real numbers such that $T_{3}(s)=T_{3}(t)$. For any integer $k$, we have

$$
\frac{T_{k}(t)-T_{k}(s)}{t-s}=\frac{2}{\sqrt{3}} \sin \frac{k \pi}{3} V_{k-1}(s+t) \text {. }
$$

Lemma B. Let $n \geq 3$ be an integer.

Let $s_{1}<s_{2}<\cdots<s_{n}$ and $t_{1}<\cdots<t_{n}$ be real numbers such that $T_{3}\left(s_{i}\right)=T_{3}\left(t_{i}\right)$. Let $u_{i}=t_{i}+s_{i}$. We have

$$
\sum_{i=1}^{n} u_{i}^{2} \leq n+4, \sum_{i=1}^{n} u_{i}^{4} \leq n+22 .
$$

\subsection{Proof of the theorem}

Proof. We shall prove this result by reducing it to a contradiction. Suppose the plane curve $\mathcal{C}$ parametrized by $x=P(t), y=Q(t)$ where $\operatorname{deg} P=3, \operatorname{deg} Q=n+1$ is a plane projection of $K_{n}$.

By translation on $t$, one can suppose that $P(t)=t^{3}-\alpha t+\beta$. If the polynomial $P$ was monotonic, $\mathcal{C}$ would have no crossings, which is absurd. Therefore $\alpha>0$. Dividing $t$ by $\rho=\sqrt{3} / \sqrt{\alpha}$, one has $P(t)=\rho^{3}\left(t^{3}-3 t\right)+\mu$. By translating the origin and scaling $x$, one can now suppose that $P(t)=t^{3}-3 t=T_{3}(t)$.

By translating the origin and scaling $y$, we can also suppose that $Q(t)$ is monic and write

$$
P(t)=T_{3}(t), \quad Q(t)=T_{n+1}(t)+a_{n} T_{n}(t)+\cdots+a_{1} T_{1}(t) .
$$

By Bézout theorem, the curve $\mathcal{C}$ has at most $(3-1)(n+1-1) / 2=n$ double points. As it has at least $n$ crossings, we see that it has exactly $n$ crossings and therefore is a minimal crossing diagram of $K_{n}$. According to the lemma 1, there exist real numbers $s_{1}<\cdots<s_{n}, t_{1}<\cdots<t_{n}, s_{i}<t_{i}$ such that $P\left(s_{i}\right)=P\left(t_{i}\right), Q\left(s_{i}\right)=Q\left(t_{i}\right)$. Let $u_{i}=t_{i}+s_{i}, 1 \leq i \leq n$. We have

$$
\frac{Q\left(t_{i}\right)-Q\left(s_{i}\right)}{t_{i}-s_{i}}=\frac{T_{n+1}\left(t_{i}\right)-T_{n+1}\left(s_{i}\right)}{t_{i}-s_{i}}+\sum_{k=1}^{n} a_{k} \frac{T_{k}\left(t_{i}\right)-T_{k}\left(s_{i}\right)}{t_{i}-s_{i}} .
$$


so by lemma $\mathbf{A}, u_{1}, \ldots, u_{n}$ are the distinct roots of the polynomial

$$
R(u)=\varepsilon_{n+1} V_{n}(u)+\sum_{k=1}^{n} a_{k} \varepsilon_{k} V_{k-1}(u),
$$

where

$$
\varepsilon_{k}=\frac{2}{\sqrt{3}} \sin \frac{k \pi}{3}
$$

Remark 2 Note that $\varepsilon_{k}=V_{k-1}(1)$ is the 6-period sequence $\varepsilon_{0}=0, \varepsilon_{1}=1, \varepsilon_{2}=1,0,-1,-1, \ldots$ We have to consider several cases.

$\triangleright$ Case $\mathbf{n} \equiv \mathbf{2} \bmod 3 . \varepsilon_{n+1}=0$ and $R(u)$ has degree at most $n-1$. This is a contradiction.

$\triangleright$ Case $\mathbf{n} \equiv \mathbf{1} \bmod \mathbf{3}$. In this case, $n \equiv 1 \bmod 6$ and $\varepsilon_{n+1}=\varepsilon_{n}=1, \varepsilon_{n-1}=0$. Thus $R(u)$ can be written as

$$
\begin{aligned}
R(u) & =V_{n}(u)+a_{n} V_{n-1}(u)-a_{n-2} V_{n-3}(u)-\cdots+a_{2} V_{1}(u)+a_{1} \\
& =u^{n}+a_{n} u^{n-1}-(n-1) u^{n-2}+\cdots .
\end{aligned}
$$

using equation 2. Therefore we get

$$
\sum_{1 \leq i \leq n} u_{i}=-a_{n}, \sum_{1 \leq i<j \leq n} u_{i} u_{j}=-(n-1)
$$

and then

$$
\sum_{i=1}^{n} u_{i}^{2}=\left(\sum_{i=1}^{n} u_{i}\right)^{2}-2 \sum_{1 \leq i<j \leq n} u_{i} u_{j}=a_{n}^{2}+2(n-1) \geq 2(n-1) .
$$

According to lemma $\mathbf{B}$ we also have $\sum_{i=1}^{n} u_{i}^{2} \leq n+4$, we get a contradiction for $n>6$.

$\triangleright$ Case $\mathbf{n} \equiv \mathbf{0} \bmod \mathbf{3}$. In this last case we have $n=3 \bmod 6$, so $\varepsilon_{n+1}=-1, \varepsilon_{n}=0$ and $\varepsilon_{n-1}=1$, so

$$
-R(u)=V_{n}(u)-a_{n-1} V_{n-2}(u)-a_{n-2} V_{n-3}(u)+\cdots-a_{2} V_{1}(u)-a_{1} .
$$

Let $\sigma_{i}$ be the coefficients of

$$
-R(u)=u^{n}+\sum_{k=1}^{n}(-1)^{k} \sigma_{k} u^{n-k} .
$$

From the equation 2, we see that

$$
\sigma_{1}=0, \sigma_{2}=-\left(a_{n-1}+n-1\right), \sigma_{4}=(n-3) a_{n-1}+\frac{(n-2)(n-3)}{2} .
$$

Let $S_{k}$ be the Newton sums $\sum_{i=1}^{n} u_{i}^{k}$ of the roots of the polynomial $R$. Using the classical Newton formulas $($ FS $)$, we obtain

$$
S_{1}=\sigma_{1}=0, S_{2}=\sigma_{1}^{2}-2 \sigma_{2}=-2 \sigma_{2}, S_{4}=2 \sigma_{2}^{2}-4 \sigma_{4},
$$

and then

$$
S_{4}=2\left(a_{n-1}+2\right)^{2}+6 n-18 \geq 6 n-18 .
$$

By the lemma $\mathbf{B}$, we deduce that $22+n \geq 6 n-18$, i.e. $n \leq 8$ so $n=3$. 


\subsection{Proof of lemmas A and B}

We shall use the following lemma

Lemma 3 (Lissajous ellipse) Let $s \neq t$ be complex numbers such that

$$
T_{3}(t)=T_{3}(s) .
$$

There exists a complex number $\alpha$ such that

$$
s=2 \cos (\alpha+\pi / 3), \quad t=2 \cos (\alpha-\pi / 3) .
$$

Furthermore, $\alpha$ is real if and only if $s$ and $t$ are both real, and then $t>s$ if and only if $\sin \alpha>0$.

Proof. We have

$$
\frac{T_{3}(t)-T_{3}(s)}{t-s}=t^{2}+s^{2}+s t-3
$$

Then, if $T_{3}(t)=T_{3}(s), t \neq s$, we get

$$
\frac{3}{2}(t+s)^{2}+\frac{1}{2}(t-s)^{2}=2\left(t^{2}+s^{2}+s t\right)=6 .
$$

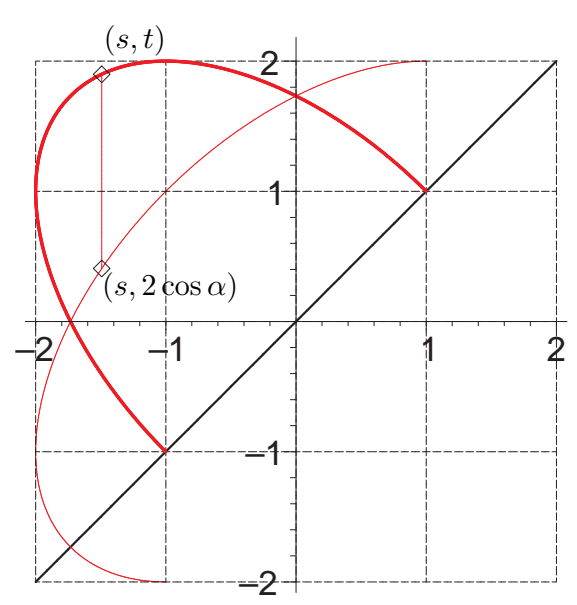

That means

$$
\left[\frac{t+s}{2}\right]^{2}+\left[\frac{t-s}{2 \sqrt{3}}\right]^{2}=1
$$

Then there exists a complex number $\alpha$ such that

$$
\cos \alpha=\frac{t+s}{2}, \sin \alpha=\frac{t-s}{2 \sqrt{3}}
$$

that is

$$
t=2 \cos (\alpha-\pi / 3), s=2 \cos (\alpha+\pi / 3) .
$$

$\alpha$ is real if and only if $\cos \alpha$ and $\sin \alpha$ are both real that is to say, iff $s$ and $t$ are real. In this case: $t>s \Leftrightarrow \sin \alpha>0$.

In order to prove lemma $\mathbf{B}$, we shall use the following lemma which describes the geometrical configuration. Let us denote $s(\alpha)=2 \cos (\alpha+\pi / 3)$ and $t(\alpha)=$ $2 \cos (\alpha-\pi / 3)$.

Lemma 4 Let $\alpha, \alpha^{\prime} \in[0, \pi]$ be such that $s(\alpha)<s\left(\alpha^{\prime}\right)$, and $t(\alpha)<t\left(\alpha^{\prime}\right)$. Then $\alpha>\alpha^{\prime}$ and $\frac{2 \pi}{3}>\frac{\alpha+\alpha^{\prime}}{2}>\frac{\pi}{3}$.

so $\alpha>\alpha^{\prime}$.

Proof. We have $2 \cos \alpha=s(\alpha)+t(\alpha)<s\left(\alpha^{\prime}\right)+t\left(\alpha^{\prime}\right)=2 \cos \alpha^{\prime}$

$$
\begin{aligned}
t\left(\alpha^{\prime}\right)-t(\alpha) & =4 \sin \left(\frac{\alpha+\alpha^{\prime}}{2}-\frac{\pi}{3}\right) \cdot \sin \left(\frac{\alpha-\alpha^{\prime}}{2}\right)>0, \\
s\left(\alpha^{\prime}\right)-s(\alpha) & =4 \sin \left(\frac{\alpha+\alpha^{\prime}}{2}+\frac{\pi}{3}\right) \cdot \sin \left(\frac{\alpha-\alpha^{\prime}}{2}\right)>0 .
\end{aligned}
$$

From $\alpha>\alpha^{\prime}$ we get $0<\frac{\alpha+\alpha^{\prime}}{2}-\frac{\pi}{3}$ and $\frac{\alpha+\alpha^{\prime}}{2}+\frac{\pi}{3}<\pi$, that is to say

$$
\frac{\pi}{3}<\frac{\alpha+\alpha^{\prime}}{2}<\frac{2 \pi}{3}
$$




\section{Proof of lemma B.}

Let $s_{1}<\cdots<s_{n}$ and $t_{1}<\cdots<t_{n}$ be such that $T_{3}\left(s_{i}\right)=T_{3}\left(t_{i}\right)$. Using lemmas 3 and 4 there are $\left.\alpha_{1}>\cdots>\alpha_{n} \in\right] 0, \pi\left[\right.$ such that $s_{i}=s\left(\alpha_{i}\right), t_{i}=t\left(\alpha_{i}\right)$ and we have

$$
\frac{2 \pi}{3}>\frac{\alpha_{1}+\alpha_{2}}{2}>\alpha_{2}>\cdots>\alpha_{n-1}>\frac{\alpha_{n-1}+\alpha_{n}}{2}>\frac{\pi}{3} .
$$

At least two of the $\alpha_{i}$ 's lie in the intervals $\left.] 0, \pi / 2\right]$ or $[\pi / 2, \pi[$. We have only two cases to consider: $\pi>\alpha_{1}>\alpha_{2} \geq \frac{\pi}{2}$, or $\frac{\pi}{2} \geq \alpha_{n-1}>\alpha_{n}>0$.

On the other hand, we get the equality

$$
\cos ^{2} x+\cos ^{2} y=1-\cos ^{2}(x+y)+2 \cos x \cos y \cos (x+y) .
$$

Case 1. $\frac{\pi}{2} \geq \alpha_{n-1}>\alpha_{n}>0$.

We get $\cos \alpha_{n} \geq 0, \cos \alpha_{n-1} \geq 0$ and $\cos \left(\alpha_{n-1}+\alpha_{n}\right)<-\frac{1}{2}$ so eq. 5 becomes

$$
\cos ^{2} \alpha_{n-1}+\cos ^{2} \alpha_{n} \leq 1-\cos ^{2}\left(\alpha_{n-1}+\alpha_{n}\right) \leq \frac{3}{4}
$$

and

$$
\begin{aligned}
\sum_{i=1}^{n} \cos ^{2} \alpha_{i} & =\cos ^{2} \alpha_{1}+\sum_{i=2}^{n-2} \cos ^{2} \alpha_{i}+\left(\cos ^{2} \alpha_{n-1}+\cos ^{2} \alpha_{n}\right) \\
& \leq 1+(n-3) \cdot \frac{1}{4}+\frac{3}{4}=\frac{1}{4}(n+4),
\end{aligned}
$$

that is

$$
S_{2}=\sum_{i=1}^{n} u_{i}^{2}=\sum_{i=1}^{n}\left(2 \cos \alpha_{i}\right)^{2} \leq n+4
$$

$\triangleright$ Case 2. $\pi>\alpha_{1}>\alpha_{2} \geq \frac{\pi}{2}$.

We get $\cos \alpha_{1} \leq 0, \cos \alpha_{2} \leq 0$ and $\cos \left(\alpha_{1}+\alpha_{2}\right)<-\frac{1}{2}$ so eq. 5 becomes

$$
\cos ^{2} \alpha_{1}+\cos ^{2} \alpha_{2} \leq 1-\cos ^{2}\left(\alpha_{1}+\alpha_{2}\right) \leq \frac{3}{4}
$$

and similarly, we get

$$
S_{2}=\sum_{i=1}^{n}\left(2 \cos \alpha_{i}\right)^{2} \leq n+4
$$

Analogously, we get $\cos ^{4} x+\cos ^{4} y \leq\left(\cos ^{2} x+\cos ^{2} y\right)^{2}$, and we deduce:

$$
S_{4}=\sum_{i=1}^{n}\left(2 \cos \alpha_{i}\right)^{4} \leq n+22 .
$$




\section{Proof of lemma A.}

Let $s<t$ be real numbers such that $T_{3}(s)=T_{3}(t)$. According to the ellipse lemma 3 , there exists a real number $\alpha$ such that

$$
t=2 \cos (\alpha-\pi / 3), \quad s=2 \cos (\alpha+\pi / 3) .
$$

We have $s+t=2 \cos \alpha$ and $t-s=4 \sin \frac{\pi}{3} \sin \alpha$, so

$$
\begin{aligned}
\frac{T_{k}(t)-T_{k}(s)}{t-s} & =\frac{2(\cos k(\alpha-\pi / 3)-\cos k(\alpha+\pi / 3))}{4 \sin \frac{\pi}{3} \sin \alpha}=\frac{\sin k \alpha \cdot \sin \frac{k \pi}{3}}{\sin \alpha \cdot \sin \frac{\pi}{3}} \\
& =\frac{2}{\sqrt{3}} \sin \frac{k \pi}{3} V_{k-1}(2 \cos \alpha) .
\end{aligned}
$$

\section{Parametrized models of $K_{3}, K_{5}, K_{7}$ and $K_{9}$}

We get parametrizations of $K_{n}: \mathcal{C}=(x(t), y(t), z(t))$, with $n$ crossings obtained for parameter values satisfying the hypothesis of lemma $\mathbf{1}$. According to lemma $\mathbf{A}$, we choose $n$ distinct points $-1 \leq u_{1}<\cdots<u_{n} \leq 1$. We look for $Q_{1}$ and $Q_{2}$ of minimal degrees, such that

$$
R_{1}(t+s)=\frac{Q_{1}(t)-Q_{1}(s)}{t-s}, R_{2}(t+s)=\frac{Q_{2}(t)-Q_{2}(s)}{t-s}
$$

satisfy, for $i=1, \ldots, n$,

$$
R_{1}\left(u_{i}\right)=0, R_{2}\left(u_{i}\right)=(-1)^{i} .
$$

We then choose $y(t)=Q_{1}(t)$ and $z(t)=Q_{2}(t)$. We also add some linear combinations of $T_{6 i}$ efficiently. We then obtain a knot whose projection is alternating, when $R_{1}$ has no more roots in $[-2,2]$. As we have chosen symmetric $u_{i}$ 's, all of our curves are symmetric with respect to the $y$-axis.

\subsection{Parametrization of $K_{3}$}

We can parametrize $K_{3}$ by $x=T_{3}(t), y=T_{4}(t), z=T_{5}(t)$. It is a Lissajous space curve (compare [Sh]). The plane curve $\left(T_{3}(t), T_{4}(t)\right)$ has 3 crossing points. The plane curve $\left(T_{3}(t), T_{5}(t)\right)$ has 4 crossing points corresponding to parameters $\left(s_{i}, t_{i}\right)$ with

$$
s_{1}<s_{2}<s_{3}<s_{4}<t_{2}<t_{1}<t_{4}<t_{3}
$$

so there do not exist real numbers $s_{1}<s_{2}<s_{3}$, and $t_{1}<t_{2}<t_{3}$ such that $x\left(s_{i}\right)=x\left(t_{i}\right), z\left(s_{i}\right)=$ $z\left(t_{i}\right)$.

This example shows that our method cannot be generalized when the projections of $K_{n}$ have at least $n+1$ crossing points. 


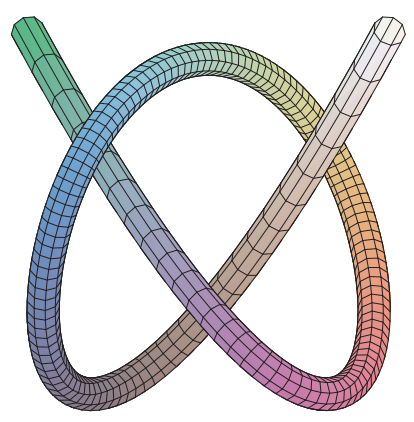

bottom view of $K_{3}$

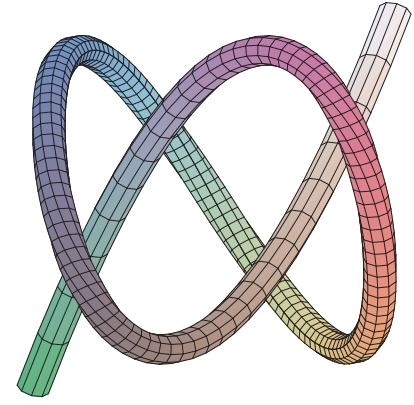

face view of $K_{3}$

Figure 4: The trefoil knot $K_{3}$

\subsection{Parametrization of $K_{5}$}

Let us consider the curve of degree $(3,7,8)$ :

$$
\begin{aligned}
& x=T_{3}(t), \\
& y=T_{8}(t)-2 T_{6}(t)+2.189 T_{4}(t)-2.170 T_{2}(t), \\
& z=T_{7}(t)-0.56 T_{5}(t)-0.01348 T_{1}(t) .
\end{aligned}
$$

The curve $(x(t), y(t))$ has exactly 5 double points when the projection $(x(t), z(t))$ has exactly 6 . Note here that $\operatorname{deg} z(t)<\operatorname{deg} y(t)$. In conclusion we have found a curve of degree $(3,7,8)$. Using

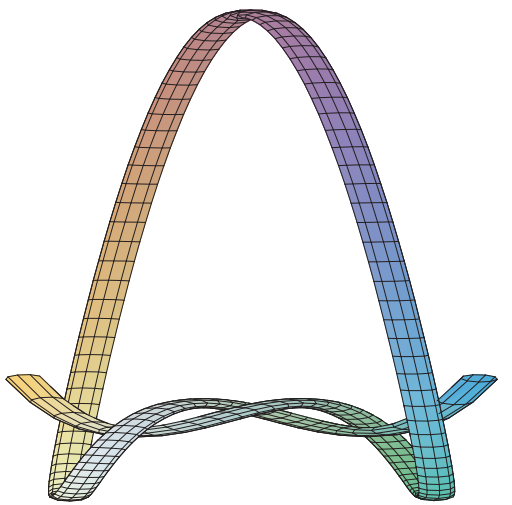

bottom view

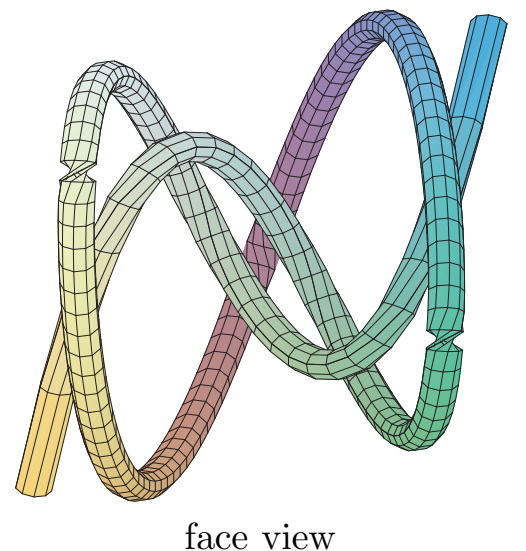

face view

Figure 5: Knot $K_{5}$

our theorem, we see that this curve has minimal degree. A. Ranjan and R. Mishra showed the existence of such an example ([RS, Mi] $)$.

\subsection{Parametrization of $K_{7}$}

We choose

$$
\begin{aligned}
& x=T_{3}(t), \\
& y=T_{10}(t)-2.360 T_{8}(t)+4.108 T_{6}(t)-6.037 T_{4}(t)+7.397 T_{2}(t), \\
& z=T_{11}(t)+3.580 T_{7}(t)-3.739 T_{5}(t)-T_{1}(t) .
\end{aligned}
$$




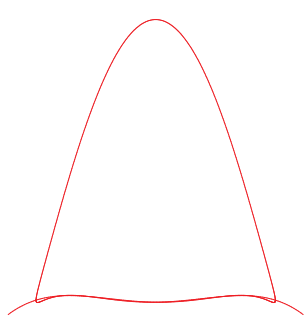

Bottom view

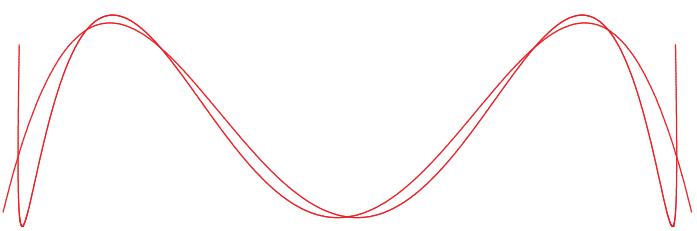

Zoom on the bottom view

Figure 6: Knot $K_{7}$

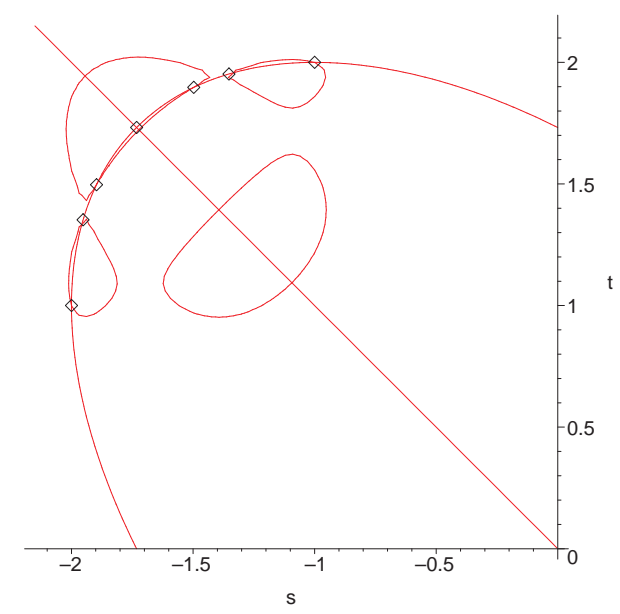

The values of the parameters corresponding to the double points are obtained as intersection points between the ellipse

$$
t^{2}+s^{2}+s t-3=0
$$

and the curve of degree 9 :

$$
(y(t)-y(s)) /(t-s)=0
$$

The curve $(x(t), y(t))$ has exactly 7 double points corresponding to $\cos (\alpha)=\{ \pm 1 / 2, \pm 3 / 10, \pm 2 / 10,0\}$.

In conclusion we have found a curve of degree $(3,10,11)$. Using our theorem, this curve has minimal degree.

\subsection{Parametrization of $K_{9}$}

We choose polynomials of degree $(3,13,14)$.

$$
\begin{aligned}
x= & T_{3}(t), \\
y= & T_{14}(t)-4.516 T_{12}(t)+12.16 T_{10}(t)-24.46 T_{8}(t)+39.92 T_{6}(t) \\
\quad & \quad-55.30 T_{4}(t)+66.60 T_{2}(t), \\
z= & T_{13}(t)-2.389 T_{11}(t)-5.161 T_{7}(t)+5.161 T_{5}(t)+1.397 T_{1}(t) .
\end{aligned}
$$

The curve $(x(t), y(t))$ has exactly 9 double points corresponding to $\cos (\alpha)=\{ \pm 1 / 2, \pm 3 / 10, \pm 2 / 10, \pm 1 / 10,0\}$. One can prove that it is minimal under the assumption that the projection $(x(t), y(t))$ has exactly 9 double points.

\section{Conclusion}

We have found minimal degree polynomial curves for torus knots $K_{n}, n=3,5,7$. For degree 9 , one can prove that it is minimal under the assumption that the projection $(x(t), y(t))$ has exactly 9 double points. We have similar constructions for higher degrees.

\section{Acknowledgments}

We would like to thank Julien Marché for fruitful discussions on knot theory. 


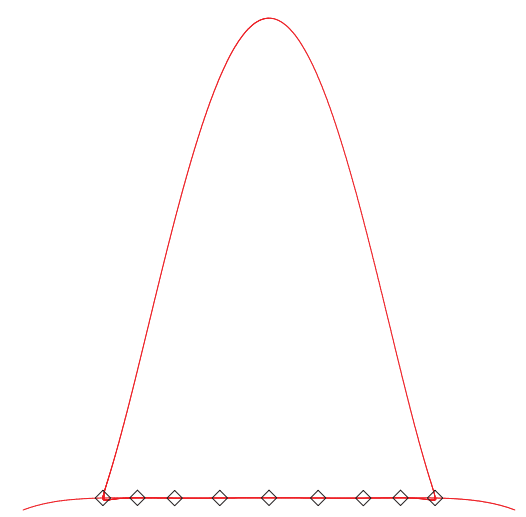

Bottom view

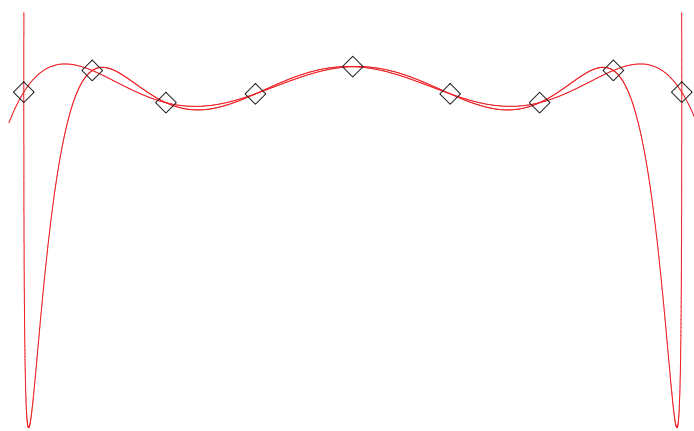

Zoom on the bottom view

Figure 7: Knot $K_{9}$

\section{References}

[FS] Faddeev, D., Sominskij, I. Problems in higher algebra, W. H. Freeman, 1965.

[Fi] G. Fischer, Plane Algebraic Curves, A.M.S. Student Mathematical Library Vol 15, 2001.

[Ka] L. Kaufmann, On knots, Princeton University Press, 1987.

[MT] W.M. Menasco, M.B. Thistlethwaite, The Tait Flyping conjecture, Bull. Amer. Math Soc. 25 (2) (1991), 403-412.

[Mi] R. Mishra, Minimal Degree Sequence for Torus knots, Journal of Knot Theory and its Ramifications, Vol. 9, No 6 (2000), 759-769.

[Mu] K. Murasugi, Jones polynomials and classical conjectures in knot theory, Topology, 26 (1987), 187-194.

[Pr] J.H. Przytycki, History of the knot theory from Vandermonde to Jones, Aportaciones Matemáticas Comunicaciones, 11 (1992), 173-195.

[RS] A. Ranjan and R. Shukla, On polynomial Representation of Torus Knots, Journal of knot theory and its ramifications, Vol. 5 (2) (1996) 279-294.

[Re] K. Reidemeister, Knot Theory, Springer-Verlag, Berlin, Heidelberg New-York, 1974.

[Sh] A.R. Shastri, Polynomial Representation of knots, Tôhoku Math. J. 44 (1992), 11-17.

[St] H. Steinhaus, Mathematical Snapshots, 3rd edition, New York: Dover 1999.

[Va] V. A. Vassiliev, Cohomology of Knot Spaces, Theory of singularities and its Applications, Advances Soviet Maths Vol 1, (1990) 